ARTICLE

DOI: $10.1038 / \mathrm{s} 41467-017-01665-3$

\title{
The activity of TRAF RING homo- and heterodimers is regulated by zinc finger 1
}

\author{
Adam J. Middleton (10 1, Rhesa Budhidarmo', Anubrita Das ${ }^{1}$, Jingyi Zhu ${ }^{1}$, Martina Foglizzo (i) ${ }^{1}$ \\ Peter D. Mace $\mathbb{D}^{1} \&$ Catherine L. Day (i) ${ }^{1}$
}

Ubiquitin chains linked through lysine63 (K63) play a critical role in inflammatory signalling. Following ligand engagement of immune receptors, the RING E3 ligase TRAF6 builds K63-linked chains together with the heterodimeric E2 enzyme Ubc13-Uev1A. Dimerisation of the TRAF6 RING domain is essential for the assembly of K63-linked ubiquitin chains. Here, we show that TRAF6 RING dimers form a catalytic complex where one RING interacts with a Ubc13 Ubiquitin conjugate, while the zinc finger 1 (ZF1) domain and linker-helix of the opposing monomer contact ubiquitin. The RING dimer interface is conserved across TRAFs and we also show that TRAF5-TRAF6 heterodimers form. Importantly, TRAF5 can provide ZF1, enabling ubiquitin transfer from a TRAF6-bound Ubc13 conjugate. Our study explains the dependence of activity on TRAF RING dimers, and suggests that both homo- and heterodimers mediated by TRAF RING domains have the capacity to synthesise ubiquitin chains.

\footnotetext{
${ }^{1}$ Department of Biochemistry, School of Biomedical Sciences, University of Otago, Dunedin 9054, New Zealand. Rhesa Budhidarmo and Anubrita Das contributed equally to this work. Correspondence and requests for materials should be addressed to C.L.D. (email: catherine.day@otago.ac.nz)
} 
M any immune signalling pathways rely on the synthesis of ubiquitin chains. Non-degradative ubiquitin chains have important roles in determining the strength, duration and type of inflammatory response by functioning as molecular glue to stabilise signalling complexes. The extent of ubiquitin chain synthesis following cytokine engagement determines whether downstream effector molecules are recruited and activated. One E3 ligase with a critical role in many immune signalling pathways is TNF receptor-associated factor 6 (TRAF6). TRAF6 was initially identified because of its requirement for interleukin1-receptor (IL1-R)-mediated activation of NF- $\mathrm{KB}^{1}$, but is now known to play key roles in multiple signalling pathways that control immunoregulatory functions ${ }^{2}, 3$. This is because ubiquitin chains that are synthesised by TRAF6 serve as a platform for the activation of downstream kinases such as TAK1 and $\mathrm{Akt}^{4,5}$.

Befitting its central role in signalling, disruption of TRAF6 function has been linked to cancer and inflammatory disorders. Amplification of TRAF6 is associated with lung carcinoma ${ }^{6}$, and poor prognosis for head and neck cancers ${ }^{7}$. Overexpression of TRAF6 predicts a poor response to chemotherapy and radiotherapy for colorectal cancer patients, with molecular studies suggesting this is because TRAF6 regulates mitochondrial translocation of $\mathrm{p} 53^{8}$. It has also been suggested that chronic activation of TRAF6 is associated with aberrant splicing in haemopoietic cells in myelodysplastic syndromes ${ }^{9}$.

TRAF6 belongs to the TRAF family of proteins (TRAF1-7), which are defined by the presence of a TRAF-C/MATH domain and a TRAF-N or coiled coil (CC) domain ${ }^{10}$. These domains are responsible for trimerisation and receptor binding ${ }^{10,11}$. At their $\mathrm{N}$-termini TRAF2-7 have a RING domain, followed by a series of zinc finger (ZF) domains ${ }^{10}$ (Fig. 1a). The RING domain is common to many ubiquitin E3 ligases and, when dimeric, confers on TRAF6 its ubiquitin chain-building activity ${ }^{12}$. In general, RING E3 ligases bring together a substrate and a ubiquitinconjugated E2 enzyme resulting in transfer of ubiquitin to a substrate lysine residue ${ }^{13,14}$. There are $\sim 30$ E2s that assemble ubiquitin chains of different types. TRAF6 preferentially builds Lys63-linked ubiquitin chains by virtue of its interaction with the Ubc13-Uev1A (also called Ube2N-Ube2V1) E2 complex following engagement of receptors ${ }^{15}$. For other TRAFs, the E2 partners are still to be defined, and in fact TRAF2 contains an insertion in its RING domain that appears to abrogate E2 binding ${ }^{16}$.

The RING domain not only recruits the E2 ubiquitin (E2 Ub) conjugate, but also enhances the rate of ubiquitin transfer from the E2 to the substrate ${ }^{17,18}$. Recent studies have shown RING E3s a

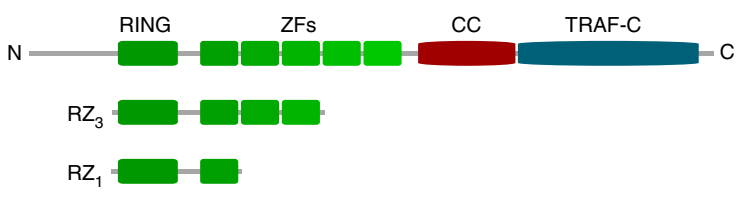

C

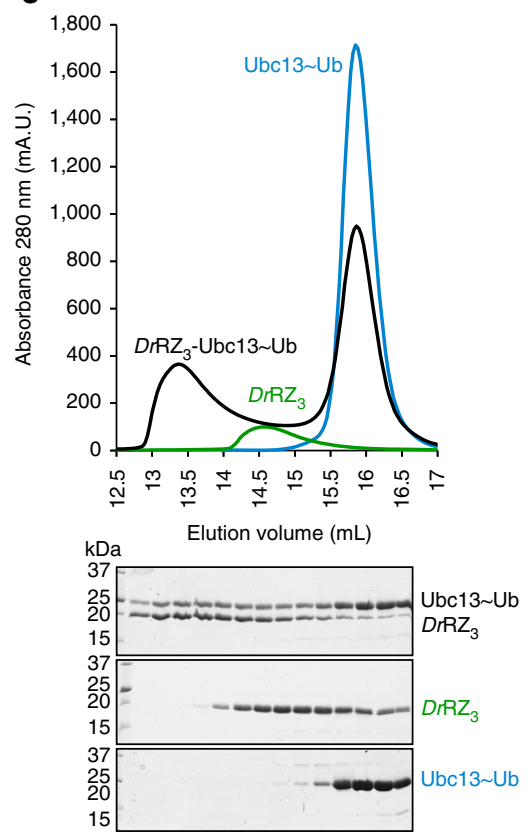

b

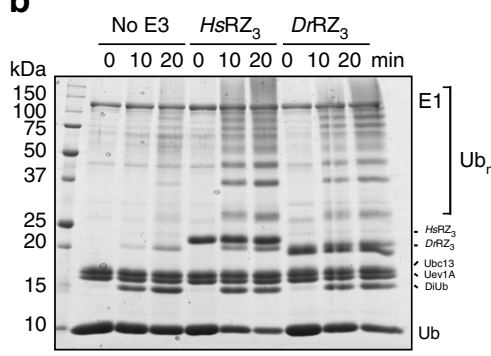

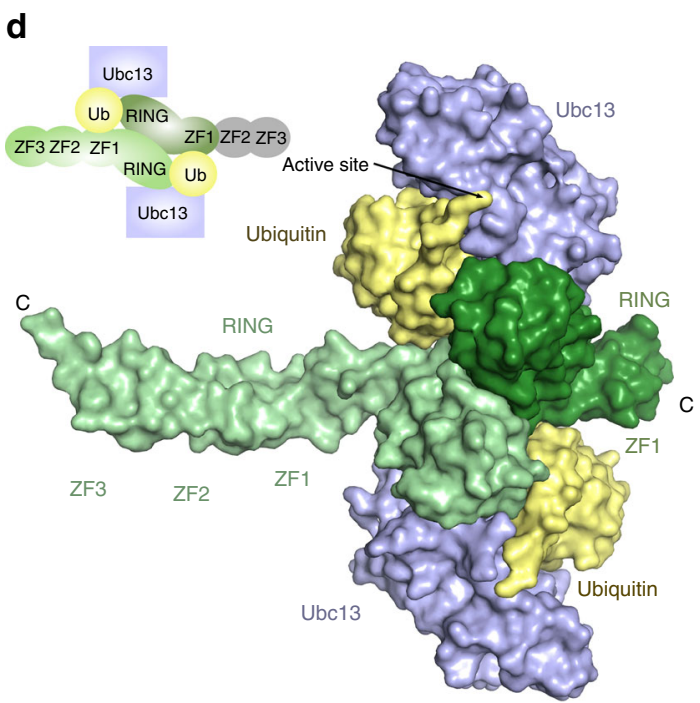

Fig. 1 Structural characterisation of TRAF6 in complex with a Ubc13 Ub conjugate. a Schematic showing the domains of TRAF6, with the RZ $Z_{3}$ and RZ 1 constructs indicated below. ZF: zinc finger, CC: coiled-coil. b Multi-turnover activity assay comparing $H$. sapiens and $D$. rerio RZ $Z_{3}$ TRAF6. Each form of TRAF6 RZ 3 was incubated with E1, Ubc13-Uev1A and ubiquitin at $37^{\circ} \mathrm{C}$ for the indicated times. c Analytical size exclusion profile of $250 \mu \mathrm{M}$ DrRZ 3 and the isopeptide-linked Ubc13-ubiquitin conjugate (Ubc13 Ub) alone, or a mixture of the two were separated using a Superdex 200 10/300 column. Equivalent fractions from each run were resolved by $16 \%$ SDS-PAGE. $\mathbf{d}$ Crystal structure of the complex of DrRZ 3 and Ubc13 Ub showing the asymmetric unit. The two TRAF6 monomers are in shades of green, ubiquitin is yellow and Ubc13 is blue. The disordered ZF2 and ZF3 are indicated in grey in the schematic 
achieve this by binding the E2 conjugated ubiquitin so that the flexible linker between ubiquitin and the E2 is bound to a groove on the $\mathrm{E} 2^{19-24}$. While some RING-ubiquitin contacts are conserved $^{14}$, the additional interactions that prime the conjugate for catalysis differ ${ }^{19,20,22-25}$. Like TRAF6, the activity of many RING domains depends on their dimerisation because one RING domain binds the $\mathrm{E} 2$, while residues from the $\mathrm{C}$ terminus of the other RING protomer make essential contacts with ubiquitin $^{19,2126}$. However, TRAF RING domains do not contain a structural equivalent to the C-terminal tail that is essential for many dimeric RING domains ${ }^{19,21}$. Therefore, the mechanism by which TRAFs stabilise the E2 Ub conjugate in the active (closed) conformation remains uncertain. Furthermore, many receptor complexes contain at least two different TRAF proteins. This raises the possibility that TRAF heterodimers could be functional - as observed for other RING E3 ligases such as MDM2/MDMX and BRCA1/BARD1 ${ }^{27,} 28$.

In TRAF6, the RING together with ZF1 is required for E3 ligase activity ${ }^{12,29}$. To identify the essential features that regulate ubiquitin chain assembly by TRAF6 we have determined structures of the RING and ZF domains of TRAF6 bound to the Ubc13 Ub conjugate in two crystal forms at 3.4 and $3.9 \AA$ A. Both structures show that the Ubc13 Ub conjugate contacts both protomers of the TRAF6 dimer. Importantly, ZF1 and the helix that connects it to the RING domain contact ubiquitin and enhance the rate of ubiquitin transfer. The structure and biochemical assays explain why RING dimerisation is required for assembly of ubiquitin chains by TRAF6, and account for the importance of the ZF domains for activity. Furthermore, we also show that the conserved RING dimer interface facilitates formation of TRAF heterodimers that are functional E3 ubiquitin ligases.

\section{Results}

Structure of the Ubc13 Ub conjugate bound to the TRAF6 dimer. To understand the molecular basis of Lys63 chain synthesis by TRAF6 we sought to obtain the structure of TRAF6 bound to the Ubc13 Ub conjugate. The structure of the $\mathrm{N}$ terminal RING domain and the first three ZFs of human TRAF6 (residues 50-211) has been reported previously ${ }^{12}$ and we initially used a comparable human construct, referred to here as $\mathrm{RZ}_{3}$ $\left(H s \mathrm{RZ}_{3}\right)$. Using GST-pulldown assays and analytical size-exclusion chromatography, we established that $H s \mathrm{RZ}_{3}$ preferentially binds to the Ubc13 Ub conjugate (Supplementary Fig. 1). Because TRAF6 $\mathrm{HsRZ}_{3}$ formed a stable complex with the isopeptide-linked Ubc13 Ub conjugate, we purified the complex and set up crystal trials. We obtained crystals of the $\mathrm{HsRZ}_{3}-\mathrm{Ubc13} \mathrm{Ub}$ complex, but despite extensive optimisation these diffracted to very low resolution. In an attempt to improve crystal quality, we expressed and purified the equivalent $\mathrm{RZ}_{3}$ construct from Danio rerio TRAF6 (DrRZ $)$. The sequence of the RING domain and first ZF are highly conserved between $D r Z_{3}$ and $\mathrm{HsRZ}_{3}$ (Supplementary Fig. 2a), and together with Ubc13 and Uev1A, $D r R_{3}$ and $H s \mathrm{RZ}_{3}$ promoted the comparable synthesis of ubiquitin chains (Fig. 1b). Additionally, $D r R Z_{3}$ formed a stable complex with the Ubc13 Ub conjugate (Fig. 1c) that could be crystallised and diffracted to $3.9 \AA$ (Table 1). The structure of the complex was readily solved by molecular replacement (Supplementary Fig. 2b).

In the structure, each asymmetric unit contains a single TRAF6 $\mathrm{RZ}_{3}$ dimer bound to two Ubc13 Ub conjugate molecules (Fig. 1d). The central RING dimer is very similar to the previously reported $H s$ TRAF6 $\mathrm{RZ}_{3}$ dimer structure ${ }^{12}$, and an overlay of the RING domains (residues 62-122) has a main-chain RMSD of $1.0 \AA$. The contacts between the conjugate and TRAF6

\begin{tabular}{|c|c|c|}
\hline & $\operatorname{DrRZ}_{3}(5 \mathrm{VO0})$ & $\operatorname{DrRZ}_{1}(5 \mathrm{VNZ})$ \\
\hline \multicolumn{3}{|l|}{ Data collection } \\
\hline Space group & $\mathrm{P}_{4}{ }_{2}{ }_{1} 2$ & $\mathrm{C} 222_{1}$ \\
\hline \multicolumn{3}{|l|}{ Cell dimensions } \\
\hline$a, b, c(\AA)$ & $181.11,181.11,97.41$ & 138.36, 170.55, 97.31 \\
\hline$\alpha, \beta, \gamma\left(^{\circ}\right)$ & $90,90,90$ & $90,90,90$ \\
\hline Resolution $(\AA)$ & $49.4-3.90(4.36-3.90)^{a}$ & $\begin{array}{l}42.3-3.40 \\
(3.68-3.40)\end{array}$ \\
\hline$R_{\text {merge }}$ & $0.167(0.637)$ & $0.119(1.43)$ \\
\hline $1 / \sigma(I)$ & $7.9(2.6)$ & $9.7(1.2)$ \\
\hline $\mathrm{CC}_{1 / 2}$ & $0.993(0.802)$ & $0.997(0.640)$ \\
\hline Completeness (\%) & $97.9(98.6)$ & $99.6(98.3)$ \\
\hline Redundancy & $4.9(4.9)$ & $7.1(7.1)$ \\
\hline \multicolumn{3}{|l|}{ Refinement } \\
\hline Resolution $(\AA)$ & 3.90 & 3.40 \\
\hline No. reflections & 14,872 & 16,020 \\
\hline$R_{\text {work }} / R_{\text {free }}$ & $0.252 / 0.299$ & $0.237 / 0.294$ \\
\hline \multicolumn{3}{|l|}{ No. of atoms } \\
\hline Protein & 5660 & 5321 \\
\hline $\operatorname{lon}\left(\mathrm{Zn}^{2+}\right)$ & 8 & 6 \\
\hline Water & 0 & 0 \\
\hline \multicolumn{3}{|l|}{$B$ factors } \\
\hline Protein & 113.0 & 159.5 \\
\hline Ligand/ion & 94.21 & 131.6 \\
\hline \multicolumn{3}{|l|}{ R.m.s. deviations } \\
\hline Bond lengths $(\AA)$ & 0.023 & 0.007 \\
\hline Bond angles $\left(^{\circ}\right)$ & 1.54 & 1.06 \\
\hline
\end{tabular}

Each structure was determined from a single crystal

${ }^{a}$ Values for the highest-resolution shell are shown in parentheses

are comparable on both sides of the dimer, but the structure is asymmetric because there is no density for ZF2 and ZF3 in one molecule of TRAF6. There was no evidence of any proteolysis, and a large solvent channel occupies the space where ZF2 and ZF3 are expected, suggesting that the two missing ZFs are highly mobile in the absence of any crystal contacts. We therefore expressed and purified a truncated construct that contained the RING and just ZF1 of $D$. rerio TRAF6 (denoted $D r R Z_{1}$ ). $\mathrm{RZ}_{1}$ and $\mathrm{RZ}_{3}$ had a comparable level of activity (Supplementary Fig. 2c) suggesting that the shorter construct contains the key determinants of activity.

TRAF6 $\mathrm{RZ}_{1}$ formed a stable complex with $\mathrm{Ubc13} \mathrm{Ub}$ that allowed purification and crystallisation of the complex. The structure was solved at $3.4 \AA$ and contains two $1: 1 \quad \operatorname{Dr} \mathrm{RZ}_{1}$ Ubc13 Ub complexes in the asymmetric unit (Supplementary Fig. 2d). Each $D r R_{1}$-Ubc13 Ub conjugate complex sits on a twofold axis and as a result the crystal contains two distinct symmetrical complexes, which have a centrally positioned TRAF6 RING dimer associated with two Ubc13 Ub conjugates (Supplementary Fig. 2e). The $\alpha$-carbons of the two symmetrical $\mathrm{RZ}_{1}$ complexes overlay with an RMSD of $0.4 \AA$, and both are similar to the $\mathrm{RZ}_{3}$ complex, with an RMSD of 0.9 and $1.5 \AA$. The contacts between proteins are very similar in all TRAF6 complexes and provide a molecular basis for understanding TRAF6 function.

Architecture of the TRAF6 catalytic complex. In the TRAF6Ubc13 Ub complex structure Ubc13 binds at the canonical surface on the RING that includes loops 1 and $2^{30}$, while the Ile36 patch of ubiquitin contacts Val108, Asp109 and Asn110 in the $\mathrm{RING}^{26}$. As well as interacting with the RING domain, ubiquitin contacts ZF1 and the connecting helix of the associated TRAF6 protomer (see below) (Fig. 1d, Supplementary Fig. 2e, f). In addition, the Ile44-centred hydrophobic patch on ubiquitin interacts with residues in $\alpha 2$ of Ubc13, and the conjugate adopts 
the 'closed conformation'26. The same contacts are seen in all complexes suggesting that the Ubc13 Ub conjugate preferentially adopts the closed conformation when bound to TRAF6. Because the conjugate contacts both RINGs in the dimer, this structure accounts for the absolute dependence of ubiquitin chain assembly on TRAF RING dimerisation ${ }^{12}$.

The C-terminal tail of ubiquitin has an extended conformation stabilised by Asp89 and Asp119 of Ubc13, but does not appear to be primed (Supplementary Fig. 2g). Two mutations (K92T and K94Q) were introduced to prevent non-specific ubiquitylation of $\mathrm{Ubc} 13$ and these probably account for the altered conformation of the tail of ubiquitin. However, the overall architecture of the TRAF6-Ubc13 Ub structure resembles the RNF4-Ubc13 Ub ${ }^{26}$ and TRIM25-Ubc13 $\mathrm{Ub}^{24}$ complexes and is compatible with the Ubc13 Ub/Mms2 complex ${ }^{31}$ (Supplementary Fig. 3). This structure therefore provides a framework for understanding the transfer of ubiquitin onto Lys63 of another ubiquitin molecule, allowing the synthesis of Lys63-linked chains by TRAF6.

Activation of the Ubc13 Ub thioester by ZF1 of TRAF6. In the TRAF6-Ubc13 Ub complex, there are close contacts between ubiquitin and two Arg residues in the linker-helix and ZF1 of the interacting protomer. The sidechain of Arg126 from the linkerhelix sits at the RING dimer interface and contacts Gly87 of the interacting RING domain, as well as the main chain of Lys33 at the base of the $\alpha$-helix in ubiquitin. Whereas, Arg147 from ZF1 packs against Asp32 of ubiquitin (Fig. 2a, b). The density for both Arg residues was relatively well-defined (Supplementary Fig. 2b, f), and these residues are conserved in TRAF6 from a number of vertebrate species (Supplementary Fig. 4a).

To determine the importance of contacts between ubiquitin and the linker-helix and ZF1, we evaluated the activity of a series of TRAF6 mutant proteins. All mutants were made in $H s R Z_{3}$ and as a result the numbering of the residues mutated differs from the structure by one residue-we have attempted to make this clear by naming residues that were shown to be important (e.g. Arg link and $\mathrm{Arg}^{\mathrm{ZF} 1}$ ). Single turnover assays where wild-type Ubc13 was first charged with Cy3-labelled ubiquitin containing a K63R mutation $\left({ }^{*} \mathrm{Ub}^{\mathrm{K} 63 \mathrm{R}}\right)$ were used to assess activity. The purified conjugate was incubated with Uev1A and ubiquitin-D77 in the presence or absence of TRAF6 variants. Fluorescence imaging was used to monitor and quantify the appearance of diubiquitin $\left({ }^{*} \mathrm{Ub}^{\left.\mathrm{K} 63 \mathrm{R}_{-} \mathrm{Ub}\right)}\right.$ over time (Fig. $2 \mathrm{c}$ ). In these assays, wild-type $\mathrm{HsRZ}_{3}$ efficiently promoted discharge of $\mathrm{Ubc13}$ and diubiquitin formation, whereas the R125A (Arg ${ }^{\text {link}) ~ m u t a n t ~ a p p e a r e d ~}$ comparable to the no E3 control (Fig. 2c, d). Mutation of R146 $\left(\mathrm{Arg}^{\mathrm{ZF} 1}\right)$ also resulted in a substantial decrease in conjugate discharge, while mutation of Ser129 on the next turn of the linker-helix, and Glu144 did not impede activity. Similar results were obtained with multi-turnover chain-building assays (Fig. 2e).

While TRAF6 $\mathrm{RZ}_{3}$ does not form a highly stable dimer ${ }^{12}$ (Supplementary Fig. 4b), RING dimerisation is essential for TRAF6 E3 ligase activity. To determine if the Arg mutants influenced TRAF6 dimerisation, we analysed each variant by size exclusion chromatography (Supplementary Fig. 4c). All mutants were comparable to wild-type, except for the Arg link mutant which eluted slightly later, suggesting that the RING dimer had been destabilised. In the crystal structure Arg link contacts ubiquitin (Supplementary Fig. 2b, f), but it seems that it is also important for dimerisation. In this way Arg link resembles the aromatic residue at the $\mathrm{C}$ terminus of the dimeric RINGs of RNF4 and ML-IAP, which play dual roles in stabilising the RING dimer and the closed conformation of the conjugate ${ }^{21,32}$. In contrast, mutation of Arg ${ }^{\mathrm{ZF} 1}$ does not destabilise the dimer, but abrogates activity, suggesting that this residue contacts ubiquitin and stabilises the closed conformation of the Ubc13 Ub conjugate. Together these data suggest that ZF1 is critical for the assembly of ubiquitin chains by TRAF6.

TRAF6 dimers with a single E2 Ub binding site are active. To further investigate the importance of ZF1 and RING dimerisation, we assessed the ability of inactive TRAF6 mutants to recover activity when mixed. In addition to the Arg $\mathrm{ZF1}$ mutant that prevents ubiquitin binding, we used a I72D/L74R (IL/DR) mutant of $\mathrm{HsRZ}_{3}$ that does not interact with Ubc13 because the E2 binding interface is disrupted ${ }^{12}$. We hypothesised that each mutant would be inactive alone, but if the two TRAF6 RING species associate, the Ubc13-binding and ubiquitin-binding sites from different mutants could co-operate to form a complete binding site for the E2 Ub conjugate.

As expected the activity of the isolated mutants was significantly impaired in both the chain building and single turnover assays (Fig. 3). However, when the E2 interface mutant was mixed with the $\mathrm{ZF1}$ mutant ( $\mathrm{Arg}^{\mathrm{ZF1}}$ ) activity was considerably increased (Fig. 3). The increased activity must originate from a population of mixed TRAF6 dimers that have a single wild-type conjugate binding site comprising the E2 binding site on one RING, and ZF1 of the interacting $\mathrm{RZ}_{3}$ molecule (as indicated in schematic in Fig. 3a, c). Together, these data suggest TRAF6 RING dimers that possess a single conjugate binding site retain activity. As a result, activity can be used to assess heterodimerisation of TRAF proteins.

TRAF RING heterodimerisation. Analysis of the sequences of the RING and ZF domains from the four most similar TRAFs (TRAF2, 3, 5 and 6) revealed an extended conserved region that comprises the RING dimer interface and the ubiquitin binding site on the linker-helix (Fig. 4a, b). The high level of similarity at the dimer interface, but relative absence of sequence conservation on the remainder of the surface, suggests that TRAF RING domain heterodimers may form. In addition, the Arg in the connecting helix is conserved in all four TRAFs, suggesting that other TRAFs might have the capacity to interact with ubiquitin.

To investigate the formation of TRAF RING heterodimers, we expressed $\mathrm{RZ}_{3}$ from TRAF5 and analysed its ability to interact with TRAF6 using size-exclusion chromatography (Fig. 4c). TRAF5 and TRAF6 eluted together and earlier than either protein alone, suggesting that they preferentially form a stable dimer. In support of the increased stability of the heterodimer the mixture appeared to have greater activity than TRAF6 alone, even though TRAF5 had diminished activity with Ubc13-Uev1A in both single- and multi-turnover assays (Fig. 4d, left).

To confirm that TRAF5-TRAF6 heterodimers were active, we assessed the ability of TRAF5 to restore the activity of the Arglink and Arg ${ }^{\mathrm{ZF} 1}$ TRAF6 mutants. Substantial discharge of Ubc13 Ub and recovery of chain-building activity was observed when

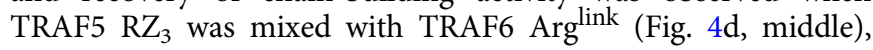
showing that TRAF5 has an intact ubiquitin binding site. This also shows that TRAF6 Arg ${ }^{\text {link }}$ retains the ability to form dimers with TRAF5 even though the mutation disrupts TRAF6 homodimers (Supplementary Fig. 4c), which is consistent with the greater stability of the TRAF5-TRAF6 heterodimers. In contrast, when the Arg in the linker-helix of TRAF5 (Arg101) was mutated (R101A, referred to as $\mathrm{RZ}_{3}$-Arg link) activity was not recovered in an equivalent mixing experiment. This is probably because, like the equivalent TRAF6 mutant, the R101A mutation destabilises dimer formation (Supplementary Fig. 4d). Together, the data suggest that Arg link plays comparable roles in both TRAF5 and TRAF6. 
a

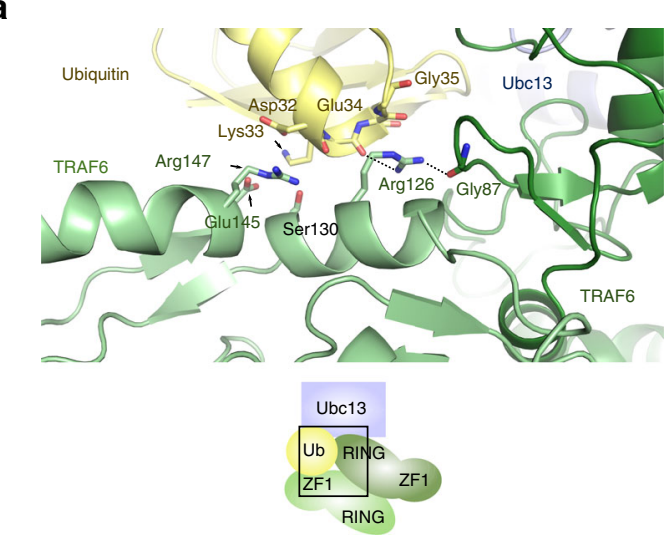

b

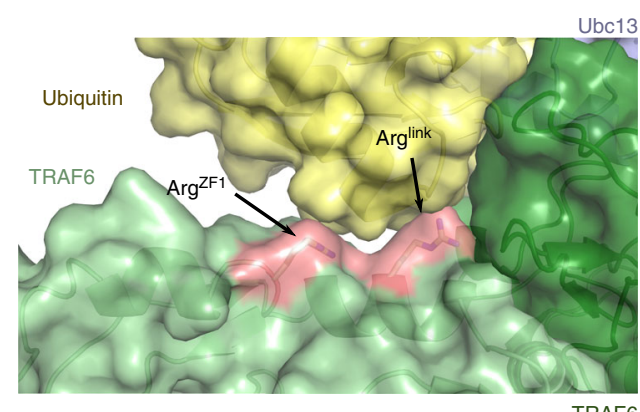

e $\frac{\text { No E3 }}{01020} \frac{\text { WT }}{1020} \frac{\text { Arglink }}{01020} \frac{\text { S129A }}{01020} \frac{\text { E144A }}{01020} \frac{\text { Arg }^{\text {ZF } 1 ~}}{01020 \mathrm{~min}}$

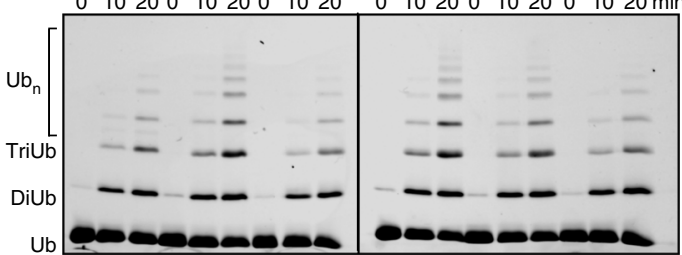

C
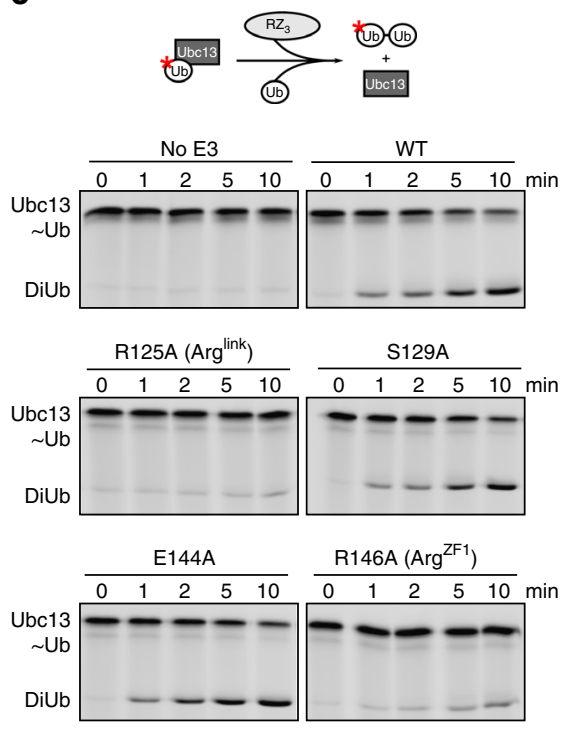

d

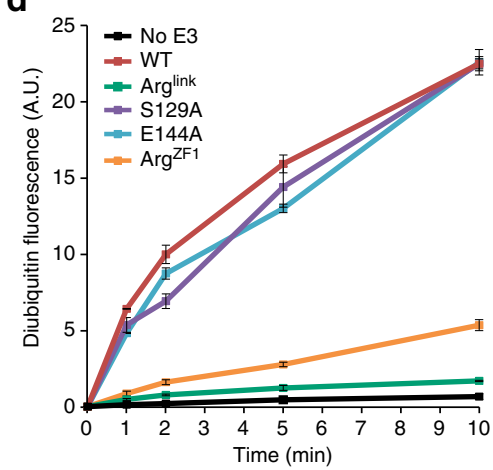

Fig. 2 The linker-helix and ZF1 of TRAF6 stabilise ubiquitin so that it is primed for attack. a Details of the molecular contacts between the RING dimer (two shades of green) and ubiquitin from the $R Z_{1}$ complex (chains $A, B$ and $C$ ). Predicted polar contacts are indicated with dashed lines. Carbon atoms coloured as in Fig. 1d, oxygen and nitrogen atoms coloured in red and blue, respectively. Numbering is as for D. rerio TRAF6. b Surface representation of the TRAF6-ubiquitin interaction. Critical residues for stabilisation are in pink. Arg126 is labelled as Arg link; Arg147 as Arg ZF1. c Ubc13 conjugated to fluorescently labelled ubiquitin was used in discharge assays in combination with no E3, wild-type $H_{s R Z}$, or mutant forms of TRAF6 as indicated. d Quantification of the formation of diubiquitin. Each experiment was performed in duplicate; error bars indicate range of measurement. e Chain-building assay using the same mutants as in panel c. Reaction mixture was spiked with $10 \%$ fluorescently labelled ubiquitin to allow visualisation of the chains

TRAF5 $\mathrm{RZ}_{3}$ also recovered activity when mixed with the TRAF6 Arg ${ }^{\mathrm{ZF}}$ mutant in both single- and multi-turnover assays (Fig. 4d, right). In TRAF5 $\operatorname{Arg}^{\mathrm{ZFI}}$ is replaced by a Gly, but the adjacent residue is an Arg (Fig. 4b). We reasoned that small adjustments in the structure of TRAF5 might allow this Arg residue to fulfil the role of $\mathrm{Arg}^{\mathrm{ZF1}}$ in TRAF6. In support of this, when TRAF5 in which Arg124 was mutated to Ala (R124A) was mixed with the TRAF6 Arg ZF1 activity was not recovered to the same extent as observed when WT TRAF5 was included.

Together these data indicate that the linker-helix and ZF1 in TRAF5 are important for activity. In addition, these results provide evidence that TRAF RING heterodimers form and promote ubiquitin transfer.

\section{Discussion}

TRAF proteins are adaptor molecules that have a trimeric 'TRAF' domain, which recognises the intracellular tails of membraneembedded cytokine receptors, and also a dimeric RING domain that assembles ubiquitin chains in response to cytokine signalling.
Here we account for the importance of TRAF RING dimers and show that the ZF1 domain of TRAF6 interacts with ubiquitin to stabilise the closed conformation of the Ubc13 Ub conjugate. Thus, ZF1 in TRAF6 plays a critical role in accelerating K63 chain synthesis by Ubc13-Uev1A. We also demonstrate that the RING domains of TRAF5 and TRAF6 form a stable heterodimer that is an active E3 ligase, supporting the notion that TRAF heterodimers can regulate ubiquitylation.

The conformation of the Ubc13 Ub conjugate bound to TRAF6 resembles that reported for the RNF4 and TRIM25 complexes with $\mathrm{Ubc} 13 \sim \mathrm{Ub}$, and is compatible with the assembly of K63-linked ubiquitin chains by TRAF6 ${ }^{24,26}$. However, even though the RING-E2 Ub interactions are conserved (Fig. 5a), the RING-RING dimer interfaces vary, and accordingly, the ubiquitin and E2 moieties bound to the distal RING are rotated relative to the proximal molecules. As a result the critical cross-dimer ubiquitin-stabilising interactions are shifted (Fig. $5 \mathrm{a}, \mathrm{b}$ ) so that in TRAF6 ubiquitin points towards the linker-helix and ZF1, where it interacts with basic residues (Arglink and $\mathrm{Arg}^{\mathrm{ZF1}}$ ). In this way, basic residues $\mathrm{C}$-terminal to the TRAF6 RING domain appear to 
a

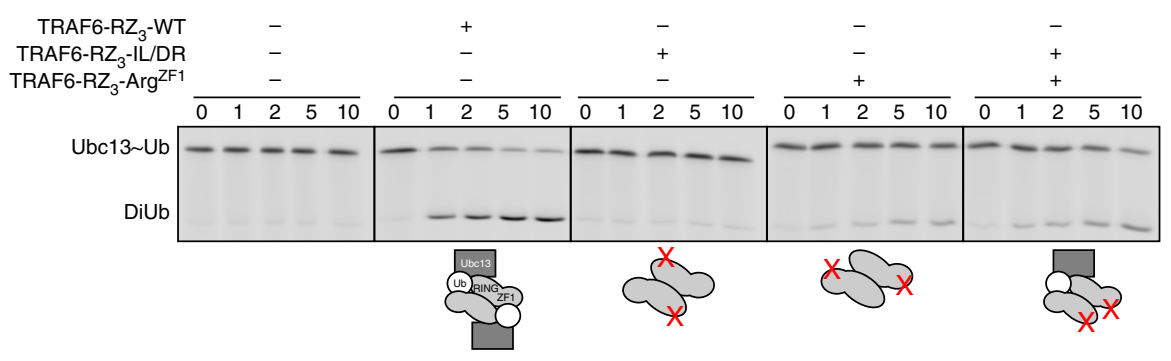

b

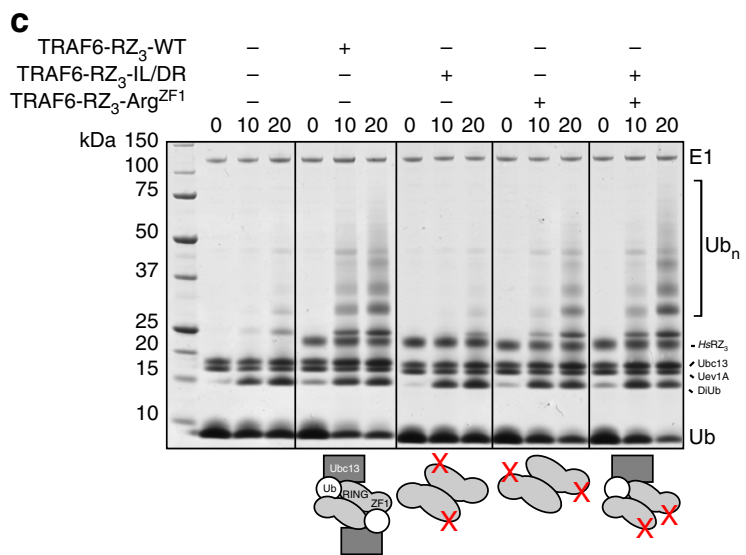

Fig. 3 TRAF6 dimers with a single conjugate binding site are active. a Single-turnover assay using fluorescently labelled conjugate performed as in Fig. 2c. Mutant proteins that disrupted either the E2 interface (IL/DR) or ubiquitin binding ( $\mathrm{Arg}^{\mathrm{ZF} 1}$ ) were incubated with the conjugate alone, or following mixing (far right). Schematics indicate the site of each mutation on TRAF6. $\mathbf{b}$ The formation of diubiquitin from panel a was quantified. Experiments were performed in duplicate; error bars represent range of measurements. c Coomassie blue stained chain-building assay using TRAF6

$\mathrm{RZ}_{3}$ mutants from panel a

fulfil the role of the well-established locking aromatic residue in other dimeric RINGs, such as RNF4 and ML-IAP ${ }^{19,} 21,26$.

A more general role for basic residues as the 'locking pin' seems likely because TRIM25 has similarly positioned residues, Lys65 and Thr67, which make comparable contacts with ubiquitin (Fig. 5b) ${ }^{23,24}$. In fact, the RING domains of TRIM25 and TRAF6 overlay remarkably well, and this similarity extends beyond the RING domain to include the TRAF6 linker-helix and the $\alpha$-helix that links the TRIM25 RING to its B-box domain. A helix

C-terminal to the RING domain is also found in the structures of other TRIMs, and in RNF125 and LNX2 (Fig. 5c) ${ }^{23,33-35}$. Each of these helices contains a basic residue in a position analogous to Arg $^{\text {link }}$ and, if dimers form, these residues could contact ubiquitin and stabilise the bound $\mathrm{E} 2 \sim \mathrm{Ub}$ conjugate in the closed conformation.

TRAF proteins function downstream of a variety of inflammatory sensors, including tumour necrosis factor (TNF), Toll-, NOD- and RIG-I-like receptors 10,36 . The specific signalling outputs from these receptors are determined by the complement of TRAF proteins recruited and the downstream effectors generated, in particular the ubiquitin chains which are built by TRAF proteins $^{2,3}, 10$. Even though Lys63-linked chains synthesised by TRAF6 have a critical role in signalling, the extent of ubiquitin chain synthesis by other TRAFs is uncertain. The E2-interacting surface of TRAFs is variable (Supplementary Fig. 5) and if E2s bind $^{16}$, it seems likely that each TRAF will bind to different E2s. However, formation of TRAF RING heterodimers, as demonstrated here, raises the possibility that even TRAFs that do not bind E2s have the potential to regulate ubiquitin transfer by providing a functional ZF1-linker-helix region to stabilise the catalytically primed complex that is recruited by a partner TRAF.
TRAF RING heterodimers have not been reported before, but multiple TRAFs are frequently found associated with signalling complexes ${ }^{10,36}$. Furthermore, the coiled coil/TRAF-C domains of TRAF1 and TRAF2 are known to form a heterotrimer comprising one copy of TRAF1 and two copies of TRAF $2^{37}$. TRAF1 does not possess a RING domain and these 1:2 TRAF1/2 heterodimers likely favour TRAF2 RING dimerisation. However, this raises the potential that other TRAF heterotrimers might form. Irrespective of whether heterotrimers form, there remains a crucial symmetry mismatch in that trimeric TRAFs interact with trimeric receptors, but RING domain dimers assemble ubiquitin chains. This symmetry mismatch is likely to be exacerbated in cells because many cytokine receptors signal via receptor clustering ${ }^{38}$, where several TRAF trimers would be expected to come into close proximity. As a result, even if TRAF homotrimers form there is considerable potential for TRAF RING heterodimers to bridge trimeric TRAF complexes at the cell membrane. For example, in the simplest case it would be anticipated that if TRAF homotrimers form and a RING dimer is favoured, one RING domain would be free to interact with the RING of a neighbouring trimer as in Fig. 6. Alternatively, if RING heterodimers are favoured it is possible that two trimers might associate to form three RING heterodimers. Other combinations are also possible and may be favoured by membrane clustering as suggested previously ${ }^{12}, 39$.

Regulation of TRAF proteins is complex, and much work remains to establish how trimeric TRAFs couple with dimeric RING domains to transduce signals. However, our data not only account for the importance of TRAF RING dimers, but also provide a mechanism by which clustering of cytokine receptors could elicit differential ubiquitin ligase activity-and the assembly of distinct ubiquitin chains from homo- and heterodimeric TRAF complexes. The nature and extent to which such signalling occurs in a cellular setting will depend on identification of the E2s bound 
a

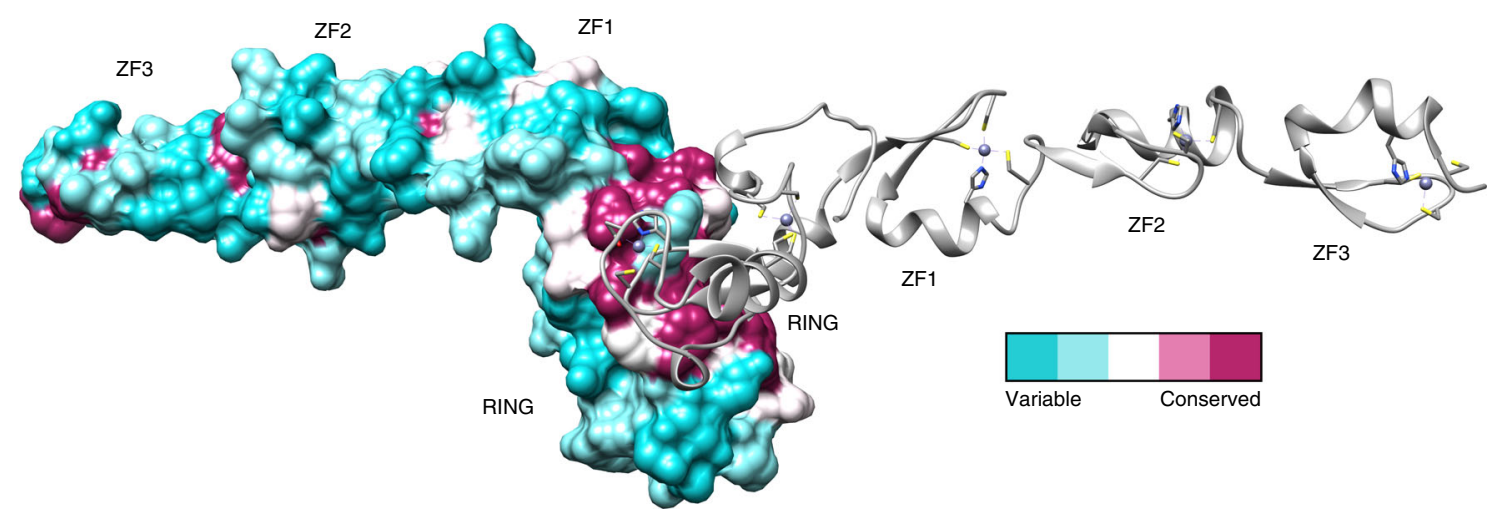

b

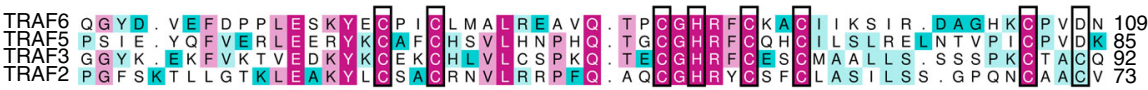
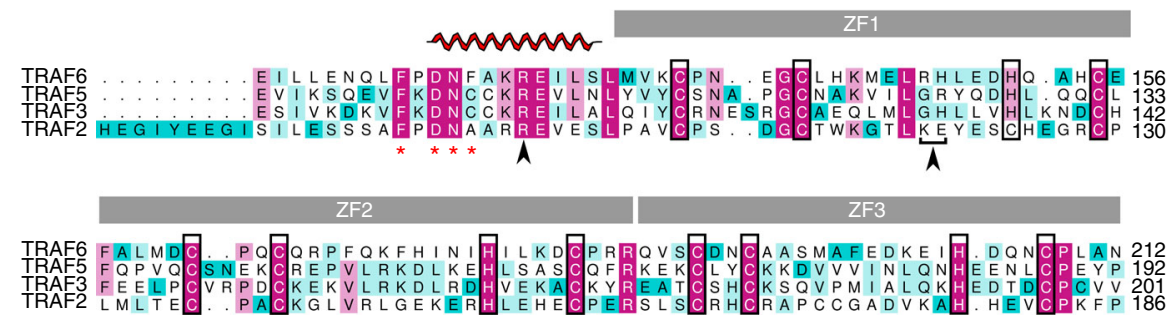

C
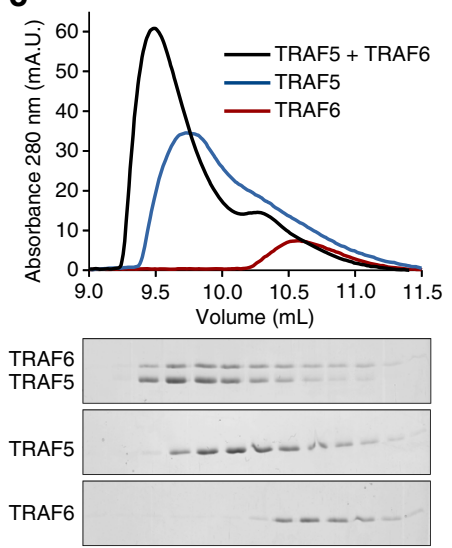

d

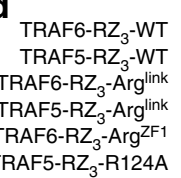
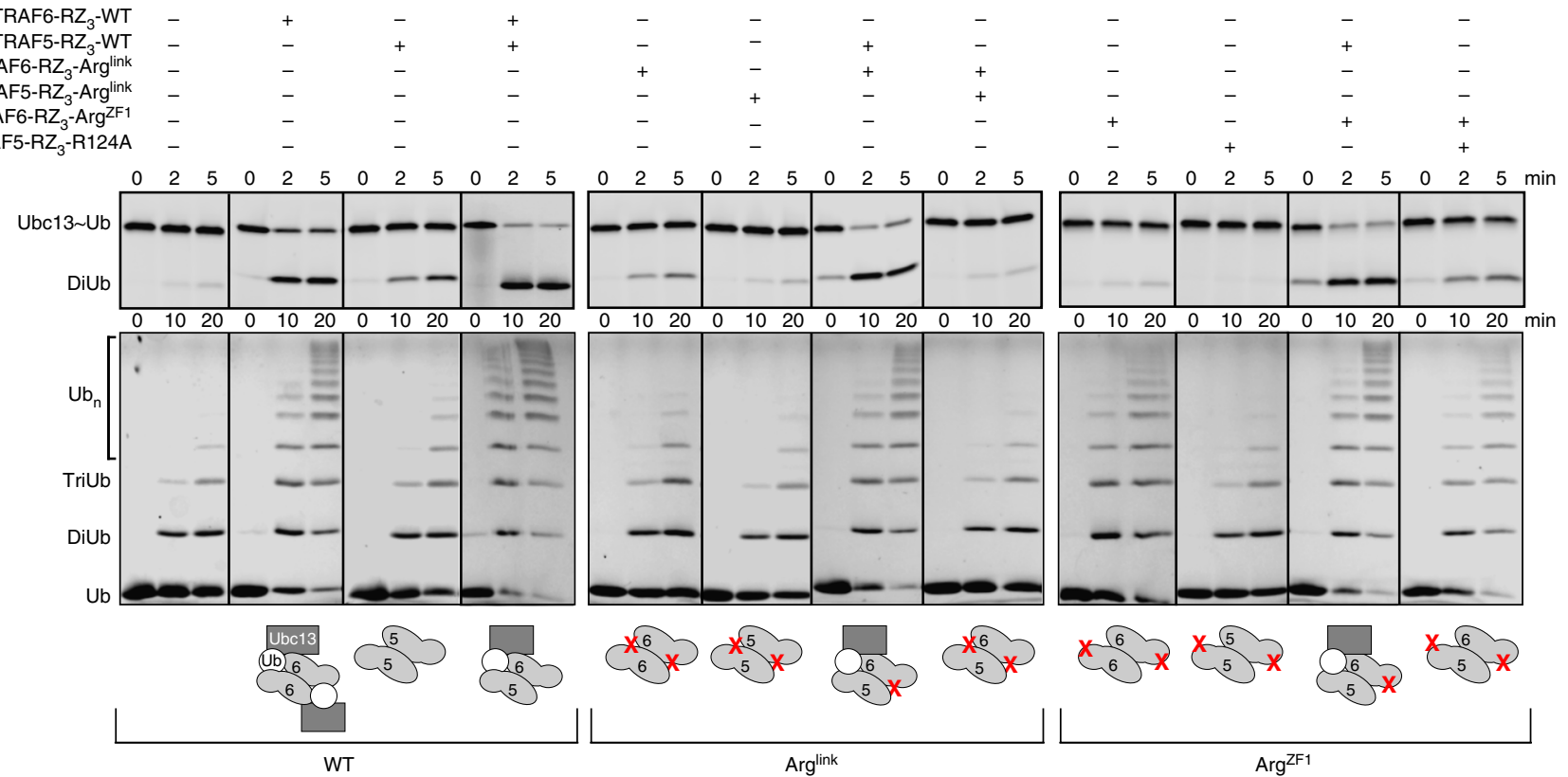

Fig. 4 The TRAF RING dimer interface is conserved allowing formation of TRAF heterodimers. a Conservation surface mapping of the RING and first three ZFs of TRAFs 2, 3, 5 and 6 mapped on the structure of TRAF6 (PDB: 3HCS) using Chimera ${ }^{51}$. Magenta represents identical residues, while cyan shows low levels of similarity. The interacting $\mathrm{RZ}_{3}$ molecule is shown in ribbon format (grey). Zinc atoms are shown as spheres, while coordinating side chains are shown as sticks with oxygen, nitrogen and sulphur atoms in red, blue and yellow, respectively. $\mathbf{b}$ Sequence alignment with identical colouring as on surface in panel a. Residues important for dimerisation are indicated by red asterisks, zinc-coordinating residues by black boxes, and the Arg residues at the ubiquitin binding interface are indicated by arrows. Domains are shown as grey boxes, and the linker-helix is indicated above the sequence. $\mathbf{c}$ Analytical size exclusion profile of TRAF6, TRAF5 and a mixture of the two. Equivalent fractions from each run were resolved by 16\% SDS-PAGE and stained with Coomassie Blue. $\mathbf{d}$ Top: discharge of fluorescently labelled Ubc13 Ub thioester conjugate following addition of TRAF6, TRAF5, mutants of each, and mixtures of these proteins as indicated. Bottom: multi-turnover assays of the same TRAF proteins using fluorescently labelled ubiquitin 
a

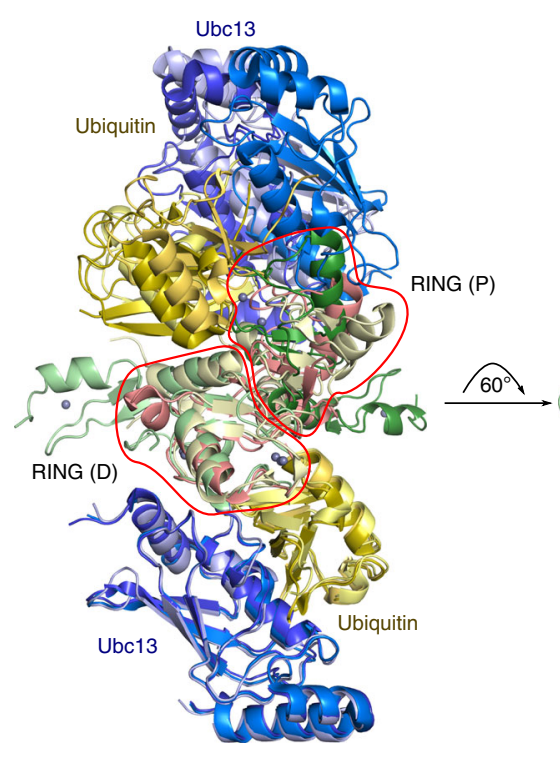

C

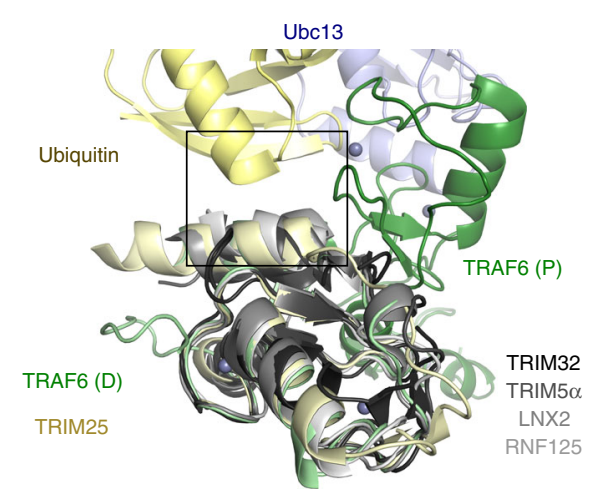

b

TRAF6

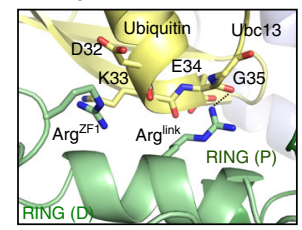

TRIM25

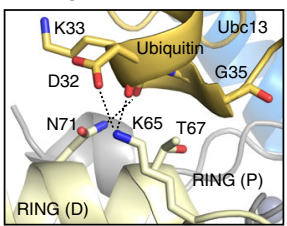

RNF4

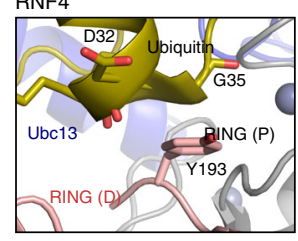

TRIM32

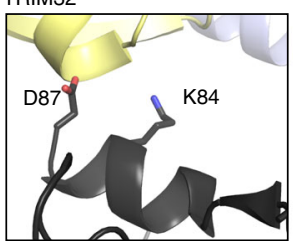

TRIM $5 \alpha$

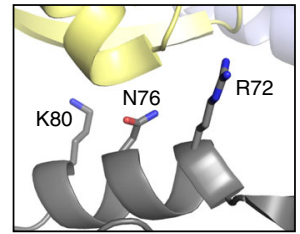

RNF125
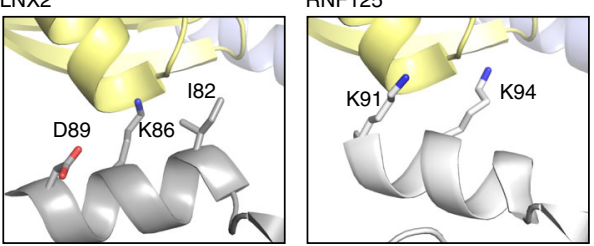

Fig. 5 Conserved interactions stabilise the position of ubiquitin in RING-Ubc13 Ub complexes. a The distal (D) RING and bound E2 Ub conjugate from TRIM25-Ubc13 Ub (PDB: 5EYA) and RNF4-Ubc13 Ub (PDB: 5AIU) were overlaid onto TRAF6-Ubc13 Ub. Zinc atoms are shown as purple spheres. (D) refers to the distal RING, (P) proximal. The RING domains are indicated with red outlines. $\mathbf{b}$ A detailed view of the contacts between the distal RING and ubiquitin that stabilise the closed conformation. Predicted hydrogen bonds are indicated by dashed lines, and sticks are coloured by atom type (oxygen, nitrogen and sulphur atoms are coloured red, blue and yellow, respectively. Carbon atoms are coloured the same as ribbon). c An overlay of four RING domains (PDBs: TRIM5 $\alpha$ 4TKP; TRIM32 5FEY; LNX2 5DIN; RNF125 5DKA) on the RING from DrRZ 3 highlighting the conserved position of the C-terminal alpha helix. On the right is a detailed view of the putative ubiquitin-gripping residues from these RING domain structures. RNF125 is modelled as a dimer, though it is monomeric in PDB 5DKA

by other TRAFs, and thus the ubiquitin chains assembled, as well as systematic analysis of TRAF RING heterodimer formation. Despite these uncertainties, heterodimeric TRAF RING domains bridging a network of trimeric TRAF proteins is an attractive mechanism to account for the complexity of signals elicited downstream of many cytokine receptors.

\section{Methods}

Protein production and purification. All proteins were expressed in Escherichia coli BL21 (DE3) (Novagen). Ubiquitin and ubiquitin variants were expressed as untagged proteins ${ }^{40}$. After overnight expression at $18^{\circ} \mathrm{C}$, the $E$. coli cells were harvested and re-suspended in $50 \mathrm{mM}$ ammonium acetate $\mathrm{pH} 4.5$ containing $1 \mathrm{mM}$ EDTA. After sonication and clarification, the supernatant was injected on a $5 \mathrm{~mL}$ HiTrap SP column (GE) and eluted with a $50 \mathrm{~mL}$ linear gradient from 0 to $1 \mathrm{M}$ $\mathrm{NaCl}$, in $50 \mathrm{mM}$ ammonium acetate $\mathrm{pH} 4.5,1 \mathrm{mM}$ EDTA. The peak fractions were pooled and injected on a HiLoad Superdex 75 16/600 column (GE) equilibrated in $20 \mathrm{mM}$ Tris- $\mathrm{HCl} \mathrm{pH} 7.5,150 \mathrm{mM} \mathrm{NaCl}$.

The human E1 protein was expressed with an N-terminal $\mathrm{His}_{6} \mathrm{tag}^{41}$. After lysis in $20 \mathrm{mM}$ Tris- $\mathrm{HCl} \mathrm{pH} \mathrm{8.5,100} \mathrm{mM} \mathrm{NaCl}, 5 \mathrm{mM}$ imidazole, the supernatant was loaded on a $5 \mathrm{~mL}$ HisTrap column. A linear $50 \mathrm{~mL}$ gradient from 5 to $250 \mathrm{mM}$ imidazole was applied. The fractions containing E1 were injected on a HiLoad Superdex 200 16/600 column (GE) equilibrated with $20 \mathrm{mM}$ Tris- $\mathrm{HCl}$ pH 7.5, 150 $\mathrm{mM} \mathrm{NaCl}$.

Homo sapiens TRAF6 constructs $\left(H s \mathrm{RZ}_{3}\right.$ : residues $50-211,20.0 \mathrm{kDa} ; H s \mathrm{RZ}_{1}$ : $50-159,13.8 \mathrm{kDa}$ ) were cloned into pET21d encoding a C-terminal His tag, while Danio rerio TRAF6 constructs $\left(D_{R} Z_{3}\right.$ : residues $50-213,19.7 \mathrm{kDa} ; \operatorname{DrRZ} Z_{1}$ : residues $50-159,13.7 \mathrm{kDa}$ ) were in pET24b. All TRAF6 constructs were induced at an O. D. $600 \mathrm{~nm}$ of 0.6 with $0.2 \mathrm{mM}$ IPTG and $0.1 \mathrm{mM} \mathrm{ZnCl}_{2}$ and incubated at $28^{\circ} \mathrm{C}$ for 4 h. Cells were sonicated in $50 \mathrm{mM}$ Tris- $\mathrm{HCl} \mathrm{pH} 7.5$ and $350 \mathrm{mM} \mathrm{NaCl}$, before being clarified and loaded into a $5 \mathrm{~mL}$ HisTrap FF column (GE). After elution, the peak fractions were loaded into a 10/300 Superdex 200 column (GE). TRAF5 was cloned into the pGEX6P3 vector containing an N-terminal GST tag. Protein expression was induced as for TRAF6, but was incubated at $18^{\circ} \mathrm{C}$ overnight. Cells were sonicated in $20 \mathrm{mM}$ Tris- $\mathrm{HCl} \mathrm{pH} \mathrm{7.5,150} \mathrm{mM} \mathrm{NaCl}$, and the lysate was clarified and bound to glutathione sepharose resin for $1 \mathrm{~h}$. The GST tag was cleaved by overnight incubation with 3C protease. The soluble fraction was loaded on a $5 \mathrm{~mL}$ HiTrap Q column, eluted with a linear $1 \mathrm{M} \mathrm{NaCl}$ gradient, and cleaned up by sizeexclusion chromatography as for TRAF6. Ubc13 and Uev1A were expressed as Nterminal GST-fusion proteins and purified using glutathione affinity, proteolysis of the GST tag, and size-exclusion chromatography.

For stable Ubc13 Ub conjugate formation, a mutated form of Ubc13 was used. This variant included C87K, K92T and K94Q mutations to allow formation of a 


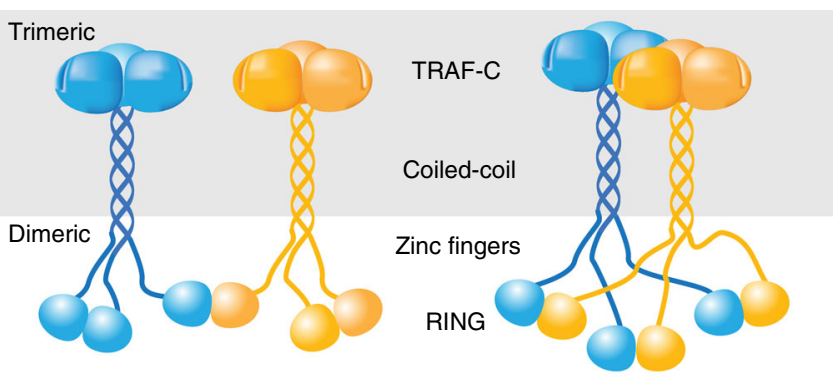

Fig. 6 Model showing how TRAF trimers might associate to enable RING dimerisation. Two potential ways that TRAF RINGs can form homo- or heterodimers that may resolve the symmetry mismatch between the trimeric TRAF-C domain and the dimeric RING domain.

stable isopeptide-linked conjugate ( $\mathrm{Ubc13} \mathrm{Ub}$ ) while disrupting formation of other species as a result of non-specific transfer from the E1. For production of isopeptide-linked conjugates, $40 \mu \mathrm{M}$ of pure Ubc13 (C87K, K92T, K94Q) was mixed with $80 \mu \mathrm{M}$ wild-type ubiquitin, $1 \mu \mathrm{M} \mathrm{E} 1,1 \mathrm{mM}$ ATP, and ATP cycling buffer containing $25 \mathrm{mM}$ Tris- $\mathrm{HCl} \mathrm{pH} 9.0,5 \mathrm{mM} \mathrm{MgCl}_{2}, 10 \mathrm{mM}$ phosphocreatine (Sigma) and $0.5 \mathrm{U} \mathrm{mL}^{-1}$ creatine phosphokinase (Sigma) ${ }^{42}$. The reaction was incubated at $37^{\circ} \mathrm{C}$ for $16 \mathrm{~h}$. The resulting Ubc13 Ub conjugate was purified using a 26/600 Superdex 75 column (GE) equilibrated in $20 \mathrm{mM}$ Tris- $\mathrm{HCl} \mathrm{pH} \mathrm{7.5,150} \mathrm{mM}$ $\mathrm{NaCl}$.

Pull-downs and analytical size-exclusion chromatography. To assess binding of TRAF6 $\mathrm{RZ}_{3}$ to Ubc13 and Ubc13 Ub conjugates, GST-fused Uev1A was mixed with TRAF6, Ubc13 and Ubc13 Ub conjugates. The incubations were performed in PBS containing $0.2 \%$ TWEEN 20 and $1 \mathrm{mM} \mathrm{DTT}$, at $4^{\circ} \mathrm{C}$ for $1 \mathrm{~h}$. Subsequently, the resin was washed three times with PBS with $0.2 \%$ TWEEN 20 before being mixed with SDS loading dye and separated by SDS PAGE. The gels were stained with Coomassie Blue.

Analytical size-exclusion chromatography (SEC) was performed by injecting $200 \mu \mathrm{L}$ of purified protein, or mixtures of proteins $\left(\mathrm{DrRZ}_{3}\right.$ and $\mathrm{Ubc13} \sim \mathrm{Ub}$ at 250 $\mu \mathrm{M} ; \mathrm{HsRZ}_{3}$ and $\mathrm{Ubcl3} / \mathrm{Ubcl3} \sim \mathrm{Ub}$ at $50 \mu \mathrm{M}$ ), over a 10/300 Superdex 200 (GE)

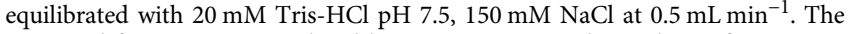
recovered fractions were analysed by SDS PAGE. For the analysis of TRAF6 proteins, each protein was injected over a 10/300 Superdex 200 Increase at $40 \mu \mathrm{M}$. For determination of the oligomeric status of TRAF6, multiangle light scattering (MALS; Dawn 8+, Wyatt) coupled to a Superdex 200 10/300 GL (GE) was used. Each TRAF5 protein was analysed at $40 \mu \mathrm{M}$ using a 10/300 Superdex 75 Increase column.

Crystallisation and structure solution. Purified D. rerio TRAF6 $\left(\mathrm{RZ}_{1}\right.$ and $\left.\mathrm{RZ}_{3}\right)$ were mixed with isopeptide-linked Ubc13 Ub conjugate for $30 \mathrm{~min}$ on ice before being separated using size-exclusion chromatography over a 10/300 Superdex 200 column (GE). The fractions corresponding to the TRAF6-Ubc13 Ub complex were pooled, concentrated to $\sim 2 \mathrm{mg} \mathrm{mL}^{-1}$, and the PACT and JCSG+ crystal screens (Molecular Dimensions) were set up with a 1:1 ratio of protein to precipitant in sitting well plates (Swissci). Trays containing $D_{r R Z}$ and $D r R Z_{1}$ resulted in crystals within $12 \mathrm{~h}$ in many conditions, which diffracted to $\sim 8 \AA$. Crystals of $D r R Z_{3}$ were grown in $100-200 \mathrm{mM} \mathrm{Na} / \mathrm{K}$ tartrate, $11-15 \%$ PEG 3350 and $100 \mathrm{mM}$ Bis-Tris propane $\mathrm{pH} 7.5 ; \mathrm{DrRZ}_{1}$ crystals were produced in $0.05-0.3 \mathrm{mM}$ sodium citrate, 100 $\mathrm{mM}$ Bis-Tris propane, and $17-23 \%$ PEG 3350. Fine tuning of the crystal conditions, changing drop ratios to 2:1 (protein: well solution), and slowing down the growth of crystals using microbatch resulted in $D r \mathrm{RZ}_{1}$ and $\mathrm{DrRZ} \mathrm{R}_{3}$ crystals that diffracted to 3.4 and $3.9 \AA$, respectively, on MX2 at the Australian Synchrotron ${ }^{43}$.

Data sets were collected for each crystal form, and processed and scaled using $\mathrm{XDS}^{44}$. Data were merged with Aimless ${ }^{45}$, and the structures were solved with Phaser-MR ${ }^{46}$ using the complex of Ubc13-TRAF6 RING domain (PDB: $3 \mathrm{HCT}^{12}$ ) followed by ubiquitin (PDB: $1 \mathrm{UBQ}^{47}$ ). For one of the $\mathrm{DrRZ}_{3}$ molecules, two of the three ZF could be automatically placed, while the third ZF had to be manually built using $\mathrm{Coot}^{48}$. Structures were refined using Refmac ${ }^{49}$, while Coot was used to iteratively build missing residues, including the C-terminal tail of ubiquitin. For $\operatorname{DrRZ}{ }_{3}, 92.1 \%$ of residues are in favoured regions, $6.6 \%$ in allowed regions and $1.3 \%$ are outliers. For $D r Z_{1}, 96.3 \%$ of residues are in favoured regions, $3.5 \%$ in allowed regions and $0.2 \%$ are outliers.

Purification of fluorescently labelled conjugate. Fluorescently tagged ubiquitin was produced by introducing a Cys residue before the beginning of the ubiquitin sequence, resulting in Met-Cys-ubiquitin. To label the free Cys purified ubiquitin was reduced with $1 \mathrm{mM}$ TCEP and mixed with $5 \mu \mathrm{L}$ of $\mathrm{Cy} 3$ for $1 \mathrm{~h}$. The sample was desalted ( $5 \mathrm{~mL}$ HiTrap desalting, GE) to remove excess dye and incubated in the dark at room temperature for $\sim 16 \mathrm{~h}$ to allow unconjugated ubiquitin to form disulphide-linked dimers. Subsequently, the mixture was separated on a $16 / 600$ HiLoad Superdex 75 column (GE) and the second peak corresponding to monomeric ubiquitin was pooled and concentrated to $\sim 2 \mathrm{mg} \mathrm{mL}^{-1}$. Thioester-linked conjugate containing fluorescent ubiquitin was produced and purified using similar conditions as before, ${ }^{50}$ except Ubc13 with a K97R mutation was mixed with ubiquitin, E1 and ATP cycling buffer at $\mathrm{pH} 7.5$. The thioester charging reaction was incubated at $37^{\circ} \mathrm{C}$ for $20 \mathrm{~min}$ before the conjugate was purified using a $10 / 300$ Superdex 75 column (GE) equilibrated with $20 \mathrm{mM}$ MES pH $6.5,150 \mathrm{mM} \mathrm{NaCl}$. The fractions containing conjugate were immediately flash frozen in liquid nitrogen.

Ubiquitylation assays. For multi-turnover ubiquitylation assays, $0.1 \mu \mathrm{M} \mathrm{E1}, 6 \mu \mathrm{M}$ Ubc13 and Uev1A, $50 \mu \mathrm{M}$ ubiquitin, and $4 \mu \mathrm{M}$ TRAF proteins (wild-type or mutants) were mixed together in a buffer containing $20 \mathrm{mM}$ Tris-HCl pH 7.5, 2 $\mathrm{mM} \mathrm{DTT}, 2 \mathrm{mM} \mathrm{ATP}, 5 \mathrm{mM} \mathrm{MgCl} 2$ and $0.1 \mathrm{M} \mathrm{NaCl}$, and incubated at $37^{\circ} \mathrm{C}$. For fluorescently labelled multi-turnover assays, the reaction mixture was spiked with $10 \%$ fluorescent ubiquitin. At the indicated times, the reactions were quenched by adding SDS-PAGE loading buffer containing $\beta$-mercaptoethanol. Proteins were separated using $16 \%$ SDS-PAGE, $10-18 \%$ gradient SDS-PAGE (homemade) or commercial (4-12\% bis-Tris SDS PAGE, Invitrogen) gels. Fluorescence was imaged using an Odyssey FC imaging system (LI-COR) at $600 \mathrm{~nm}$ with a $2 \mathrm{~min}$ exposure. Gels were stained with Coomassie blue.

For single-turnover discharge assays, $10 \mu \mathrm{M}$ purified thioester linked Ubc13 Ub ${ }^{\mathrm{K} 63 \mathrm{R}}$ conjugate was mixed with $15 \mu \mathrm{M}$ Uev1A, $50 \mu \mathrm{M}$ D77 ubiquitin (a ubiquitin variant where the $\mathrm{C}$-terminal Gly is mutated to Asp to prevent charging by E1), and $4 \mu \mathrm{M}$ TRAF proteins and incubated at 20 or $37^{\circ} \mathrm{C}$ for the indicated time. The final $\mathrm{pH}$ of the discharge reactions was 7.0. Reactions were quenched with non-reducing SDS-PAGE loading dye containing $10 \mathrm{mM} \mathrm{N}$-ethylmaleimide. For quantification of fluorescently labelled conjugate, gels were scanned as for the multi-turnover assays and the intensity of fluorescent bands was quantified using Image Studio Lite (LI-COR).

Data availability. The coordinates and structure factors for TRAF6 RZ3 and RZ1 complexes with Ubc13 Ub have been deposited to the Protein Data Bank under the accession codes $5 \mathrm{VO}$ and $5 \mathrm{VNZ}$, respectively. All other data are available from the corresponding authors upon reasonable request.

Received: 30 May 2017 Accepted: 6 October 2017 Published online: 27 November 2017

\section{References}

1. Cao, Z., Xiong, J., Takeuchi, M., Kurama, T. \& Goeddel, D. V. TRAF6 is a signal transducer for interleukin-1. Nature 383, 443-446 (1996).

2. Walsh, M. C., Lee, J. \& Choi, Y. Tumor necrosis factor receptor-associated factor 6 (TRAF6) regulation of development, function, and homeostasis of the immune system. Immunol. Rev. 266, 72-92 (2015).

3. Zinngrebe, J., Montinaro, A., Peltzer, N. \& Walczak, H. Ubiquitin in the immune system. EMBO Rep. 15, 28-45 (2013).

4. Wang, C. et al. TAK1 is a ubiquitin-dependent kinase of MKK and IKK. Nature 412, 346-351 (2001).

5. Yang, W.-L. et al. The E3 ligase TRAF6 regulates Akt ubiquitination and activation. Science 325, 1134-1138 (2009).

6. Starczynowski, D. T. et al. TRAF6 is an amplified oncogene bridging the RAS and NF-kappaB pathways in human lung cancer. J. Clin. Invest. 121, 4095-4105 (2011).

7. Feng, H. et al. EGFR phosphorylation of DCBLD2 recruits TRAF6 and stimulates AKT-promoted tumorigenesis. J. Clin. Invest. 124, 3741-3756 (2014).

8. Zhang, X. et al. TRAF6 restricts p53 mitochondrial translocation, apoptosis, and tumor suppression. Mol. Cell 64, 803-814 (2016).

9. Fang, J. et al. Ubiquitination of hnRNPA1 by TRAF6 links chronic innate immune signaling with myelodysplasia. Nat. Immunol. 18, 236-245 (2017).

10. Xie, P. TRAF molecules in cell signaling and in human diseases. J. Mol. Signal. 8, 7 (2013).

11. Park, Y. C., Burkitt, V., Villa, A. R., Tong, L. \& Wu, H. Structural basis for self-association and receptor recognition of human TRAF2. Nature 398 , 533-538 (1999).

12. Yin, Q. et al. E2 interaction and dimerization in the crystal structure of TRAF6. Nat. Struct. Mol. Biol. 16, 658-666 (2009).

13. Berndsen, C. E. \& Wolberger, C. New insights into ubiquitin E3 ligase mechanism. Nat. Struct. Mol. Biol. 21, 301-307 (2014).

14. Buetow, L. \& Huang, D. T. Structural insights into the catalysis and regulation of E3 ubiquitin ligases. Nat. Rev. Mol. Cell Biol. 17, 626-642 (2016). 
15. Deng, L. et al. Activation of the IкB kinase complex by TRAF6 requires a dimeric ubiquitin-conjugating enzyme complex and a unique polyubiquitin chain. Cell 103, 351-361 (2000).

16. Yin, Q., Lamothe, B., Darnay, B. \& Wu, H. Structural basis for the lack of E2 interaction in the RING domain of TRAF2. Biochemistry 48, 10558-10567 (2009).

17. Metzger, M. B., Pruneda, J. N., Klevit, R. E. \& Weissman, A. M. RING-type E3 ligases: master manipulators of E2 ubiquitin-conjugating enzymes and ubiquitination. Biochim. Biophys. Acta 1843, 47-60 (2014).

18. Zheng, N. \& Shabek, N. Ubiquitin ligases: structure, function, and regulation. Annu. Rev. Biochem. https://doi.org/10.1146/annurev-biochem-060815-014922 (2017).

19. Plechanovova, A., Jaffray, E. G., Tatham, M. H., Naismith, J. H. \& Hay, R. T. Structure of a RING E3 ligase and ubiquitin-loaded E2 primed for catalysis. Nature 489, 115-120 (2012).

20. Dou, H., Buetow, L., Sibbet, G. J., Cameron, K. \& Huang, D. T. Essentiality of a non-RING element in priming donor ubiquitin for catalysis by a monomeric E3. Nat. Struct. Mol. Biol. 20, 982-986 (2013).

21. Dou, H., Buetow, L., Sibbet, G. J., Cameron, K. \& Huang, D. T. BIRC7-E2 ubiquitin conjugate structure reveals the mechanism of ubiquitin transfer by a RING dimer. Nat. Struct. Mol. Biol. 19, 876-883 (2012).

22. Buetow, L. et al. Activation of a primed RING E3-E2-ubiquitin complex by non-covalent ubiquitin. Mol. Cell 58, 297-310 (2015).

23. Koliopoulos, M. G., Esposito, D., Christodoulou, E., Taylor, I. A. \& Rittinger, K. Functional role of TRIM E3 ligase oligomerization and regulation of catalytic activity. EMBO. J. 35, 1204-1218 (2016).

24. Sanchez, J. G. et al. Mechanism of TRIM25 catalytic activation in the antiviral RIG-I pathway. Cell Rep. 16, 1315-1325 (2016).

25. Wright, J. D., Mace, P. D. \& Day, C. L. Secondary ubiquitin-RING docking enhances Arkadia and Ark2C E3 ligase activity. Nat. Struct. Mol. Biol. 23, 45-52 (2016).

26. Branigan, E., Plechanovova, A., Jaffray, E. G., Naismith, J. H. \& Hay, R. T. Structural basis for the RING-catalyzed synthesis of K63-linked ubiquitin chains. Nat. Struct. Mol. Biol. 22, 597-602 (2015).

27. Linke, K. et al. Structure of the MDM2/MDMX RING domain heterodimer reveals dimerization is required for their ubiquitylation in trans. Cell Death Differ. 15, 841-848 (2008)

28. Brzovic, P. S., Rajagopal, P., Hoyt, D. W., King, M. C. \& Klevit, R. E. Structure of a BRCA1-BARD1 heterodimeric RING-RING complex. Nat. Struct. Biol. 8 833-837 (2001)

29. Lamothe, B. et al. The RING domain and first zinc finger of TRAF6 coordinate signaling by interleukin-1, lipopolysaccharide, and RANKL. J. Biol. Chem. 283, 24871-24880 (2008)

30. Budhidarmo, R., Nakatani, Y. \& Day, C. L. RINGs hold the key to ubiquitin transfer. Trends Biochem. Sci. 37, 58-65 (2012).

31. Eddins, M. J., Carlile, C. M., Gomez, K. M., Pickart, C. M. \& Wolberger, C. Mms2-Ubc13 covalently bound to ubiquitin reveals the structural basis of linkage-specific polyubiquitin chain formation. Nat. Struct. Mol. Biol. 13, 915-920 (2006)

32. Plechanovova, A. et al. Mechanism of ubiquitylation by dimeric RING ligase RNF4. Nat. Struct. Mol. Biol. 18, 1052-1059 (2011).

33. Bijlmakers, M. J. et al. A C2HC zinc finger is essential for the RING-E2 interaction of the ubiquitin ligase RNF125. Sci. Rep. 6, 29232 (2016).

34. Nayak, D. \& Sivaraman, J. Structural basis for the indispensable role of a unique zinc finger motif in LNX2 ubiquitination. Oncotarget 6, 34342-34357 (2015).

35. Yudina, Z. et al. RING dimerization links higher-order assembly of TRIM5 $\alpha$ to synthesis of K63-Linked polyubiquitin. Cell Rep. 12, 788-797 (2015).

36. Häcker, H., Tseng, P.-H. \& Karin, M. Expanding TRAF function: TRAF3 as a tri-faced immune regulator. Nat. Rev. Immunol. 11, 457-468 (2011).

37. Zheng, C., Kabaleeswaran, V., Wang, Y., Cheng, G. \& Wu, H. Crystal structures of the TRAF2: CIAP2 and the TRAF1: TRAF2: cIAP2 complexes: affinity, specificity, and regulation. Mol. Cell 38, 101-113 (2010).

38. Dustin, M. L. \& Groves, J. T. Receptor signaling clusters in the immune synapse. Annu. Rev. Biophys. 41, 543-556 (2012).

39. Graves, J. D. et al. Apo2L/TRAIL and the death receptor 5 agonist antibody AMG 655 cooperate to promote receptor clustering and antitumor activity. Cancer Cell 26, 177-189 (2014).

40. Sato, Y. et al. Structural basis for specific cleavage of Lys 63-linked polyubiquitin chains. Nature $\mathbf{4 5 5}, 358-362$ (2008).
41. Berndsen, C. E. \& Wolberger, C. A spectrophotometric assay for conjugation of ubiquitin and ubiquitin-like proteins. Anal. Biochem. https://doi.org/10.1016/j. ab.2011.06.034 (2011).

42. Middleton, A. J., Budhidarmo, R. \& Day, C. L. Use of E2 ubiquitin conjugates for the characterization of ubiquitin transfer by RING E3 ligases such as the inhibitor of apoptosis proteins. Methods Enzymol. 545, 243-263 (2014).

43. McPhillips, T. M. et al. Blu-ice and the distributed control system: software for data acquisition and instrument control at macromolecular crystallography beamlines. J. Synchrotron. Radiat. 9, 401-406 (2002).

44. Kabsch, W. XDS. Acta Crystallogr. D 66, 125-132 (2010).

45. Winn, M. D. et al. Overview of the CCP4 suite and current developments. Acta Crystallogr. D 67, 235-242 (2011).

46. McCoy, A. J. et al. Phaser crystallographic software. J. Appl. Crystallogr. 40 658-674 (2007).

47. Vijay-Kumar, S., Bugg, C. E. \& Cook, W. J. Structure of ubiquitin refined at 1.8 A resolution. J. Mol. Biol. 194, 531-544 (1987).

48. Emsley, P., Lohkamp, B., Scott, W. G. \& Cowtan, K. Features and development of Coot. Acta Crystallogr. D 66, 486-501 (2010).

49. Murshudov, G., Vagin, A. \& Dodson, E. Refinement of macromolecular structures by the maximum-likelihood method. Acta Crystallogr. D 53, 240-255 (1997).

50. Foglizzo, M., Middleton, A. J. \& Day, C. L. Structure and function of the RING domains of RNF20 and RNF40, dimeric E3 ligases that monoubiquitylate histone H2B. J. Mol. Biol. 428, 4073-4086 (2016).

51. Pettersen, E. F. et al. UCSF Chimera--a visualization system for exploratory research and analysis. J. Comput. Chem. 25, 1605-1612 (2004).

\section{Acknowledgements}

We thank the New Zealand synchrotron group for facilitating access to the MX beamlines at the Australian Synchrotron, Victoria, Australia. A.D. acknowledges the support of a University of Otago Doctoral Scholarship. R.B. was supported by a Health Science Careers Development Award of the University of Otago. P.D.M. was supported by a Rutherford Discovery Fellowship from the Royal Society of New Zealand. This research was supported by funding from the Genesis Oncology Trust (NZ) and the Health Research Council of New Zealand.

\section{Author contributions}

A.J.M. performed all structure determination and analysis. R.B. initiated the study. A.D., M.F. and J.Z. completed the assays and biochemical experiments. All authors analysed data. C.L.D. supervised this study and wrote the manuscript with A.J.M. and P.D.M.

\section{Additional information}

Supplementary Information accompanies this paper at doi:10.1038/s41467-017-01665-3.

Competing interests: The authors declare no competing financial interests.

Reprints and permission information is available online at http://npg.nature.com/ reprintsandpermissions/

Publisher's note: Springer Nature remains neutral with regard to jurisdictional claims in published maps and institutional affiliations.

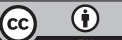

Open Access This article is licensed under a Creative Commons Attribution 4.0 International License, which permits use, sharing, adaptation, distribution and reproduction in any medium or format, as long as you give appropriate credit to the original author(s) and the source, provide a link to the Creative Commons license, and indicate if changes were made. The images or other third party material in this article are included in the article's Creative Commons license, unless indicated otherwise in a credit line to the material. If material is not included in the article's Creative Commons license and your intended use is not permitted by statutory regulation or exceeds the permitted use, you will need to obtain permission directly from the copyright holder. To view a copy of this license, visit http://creativecommons.org/ licenses/by/4.0/.

(C) The Author(s) 2017 